\title{
Chiral quark model with relativistic kinematics
}

\author{
H. Garcilazo* and A. Valcarce \\ Grupo de Física Nuclear, Universidad de Salamanca, \\ E-37008 Salamanca, Spain
}

\begin{abstract}
The non-strange baryon spectrum is studied within a three-body model that incorporates relativistic kinematics. We found that the combined effect of relativistic kinematics together with the pion exchange between quarks is able to reverse the order of the first positive- and negative-parity nucleon excited states as observed experimentally. Including the chiral partner of the pion (the $\sigma$ meson) leads to an overall good description of the spectrum.
\end{abstract}

12.39.Jh,14.20.-c

Typeset using REVTEX

*Permanent address: Escuela Superior de Física y Matemáticas, Instituto Politécnico Nacional, Edificio 9, 07738 México Distrito Federal, Mexico 
The effect of relativity in the bound state problem of three quarks is expected not to be negligible since the constituent mass of the light quarks $(u$ and $d)$ is of the same size or even smaller than the excitations observed in the baryon spectrum. Therefore, the first step towards a fully relativistic theory of the three-quark problem should be to consider relativistic kinematics which in turn requires the solution of the Faddeev equations for the three-body problem in momentum space.

The method to solve the Faddeev equations in momentum space when the interaction between quarks includes a confining potential has been recently described [1]. In particular, the problem of three quarks interacting via a one-gluon-exchange (OGE) potential plus a linear confining interaction has been studied using both the nonrelativistic Faddeev equations and a version of these equations with fully relativistic kinematics. The most important result obtained was that the relativistic kinematics improves the relative position of the positiveand negative-parity nucleon excited states $[2,3]$ as compared to the results obtained by means of the same potential in a nonrelativistic description [4]. However, the complete inversion that is observed experimentally is not achieved.

The so-called level ordering problem in the nucleon spectrum can be easily understood in the pure harmonic limit. The $N^{*}(1440) J^{P}=1 / 2^{+}$belongs to the $\left[56,0^{+}\right] S U(6)_{F S} \times O(3)$ irreducible representation and it appears in the $N=2$ band, while the $N^{*}(1535) J^{P}=1 / 2^{-}$ belongs to the $\left[70,1^{-}\right]$appearing in the $N=1$ band. As a consequence, the $N^{*}(1440)$ has $2 \hbar \omega$ energy excitation while the $N^{*}(1535)$ has only $1 \hbar \omega$ energy excitation, opposite to the order observed experimentally. Theoretically, this situation has been cured by means of appropriate phenomenological interactions as has been the case of anharmonic terms [5], scalar three-body forces [6] or pseudoscalar interactions [7,8].

The understanding of the few body problem in terms of interactions between quarks requires a more complicated dynamics than the one provided by the OGE. In particular, as dictated by the breaking of chiral symmetry a Goldstone boson exchange interaction between quarks will appear. In an effective chiral symmetric description of the baryon spectrum to lowest order a pseudoscalar one-pion-exchange (OPE) interaction between quarks is present. Such interaction is known to be very important, responsible among other things for the longrange part of the nucleon-nucleon potential [9]. Along with the pseudoscalar boson chiral symmetry requires an accompanying scalar meson field to complete the chiral multiplet. This also contributes an effective attractive interaction spin and flavor-independent, the $\sigma$ meson exchange. Such a model provides with a reasonable description of the two and three non-strange baryon systems $[9,10]$.

We have already studied the baryon spectrum by means of a dynamical model that considers Goldstone boson exchanges between quarks besides the standard OGE. In Ref. [7] we presented calculations for the baryon spectrum performed in momentum space but with a nonrelativistic kinematics. In this paper we want to focus our attention on the effect of the relativistic kinematics for the description of the low-energy baryon spectrum. In particular, the possible lowering of the first radial nucleon excitation with respect to the $L=1$ state $[2,3]$. Besides, we want to study the enhancement of this effect due to the flavor-spin structure of the pseudoscalar boson-exchange interaction. For this purpose we will make use of a quite standard quark-quark interaction composed of a confining potential, a one-gluon exchange, and Goldstone-boson exchanges.

Let us briefly resume the most important aspects of the solution of the Schrödinger 
equation with relativistic kinematics. If one replaces in the Schrödinger equation the nonrelativistic kinetic energy operator by the corresponding relativistic expression it becomes

$$
\left|\psi>=G_{0}\left(W_{0}\right)\left[V_{1}+V_{2}+V_{3}\right]\right| \psi>
$$

where if one assumes that the three particles are in the c.m. system, i.e.,

$$
\vec{k}_{1}+\vec{k}_{2}+\vec{k}_{3}=0
$$

then $W_{0}$ is the invariant mass of the system and

$$
G_{0}\left(W_{0}\right)=\frac{1}{W_{0}-\omega_{1}\left(k_{1}\right)-\omega_{2}\left(k_{2}\right)-\omega_{3}\left(k_{3}\right)},
$$

with

$$
\omega_{i}\left(k_{i}\right)=\sqrt{m_{i}^{2}+k_{i}^{2}}
$$

Making the Faddeev decomposition

$$
|\psi>=| \phi_{1}>+\left|\phi_{2}>+\right| \phi_{3}>
$$

one obtains the Faddeev equations

$$
\mid \phi_{i}>=G_{0}\left(W_{0}\right) t_{i}\left(W_{0}\right)\left[\left|\phi_{j}>+\right| \phi_{k}>\right]
$$

with

$$
t_{i}\left(W_{0}\right)=V_{i}+V_{i} G_{0}\left(W_{0}\right) t_{i}\left(W_{0}\right) .
$$

Our basis states are $\mid \vec{p}_{i} \vec{q}_{i}>$ where $\vec{p}_{i}$ is the relative momentum of the pair $j k$ measured in the c.m. frame of the pair (that is, the frame in which particle $j$ has momentum $\vec{p}_{i}$ and particle $k$ has momentum $-\vec{p}_{i}$ ) and $\vec{q}_{i}=-\vec{k}_{i}$ is the relative momentum between the pair $j k$ and particle $i$ measured in the three-body c.m. frame (that is, the frame in which the pair $j k$ has total momentum $\vec{q}_{i}$ and particle $i$ has momentum $-\vec{q}_{i}$ ). The total energy of the three particles $\omega_{1}\left(k_{1}\right)+\omega_{2}\left(k_{2}\right)+\omega_{3}\left(k_{3}\right)$ can be written in terms of the relative momenta $\vec{p}_{i}$ and $\vec{q}_{i}$ as

$$
\omega_{1}\left(k_{1}\right)+\omega_{2}\left(k_{2}\right)+\omega_{3}\left(k_{3}\right)=W_{i}\left(p_{i} q_{i}\right)+\omega_{i}\left(q_{i}\right),
$$

where

$$
W_{i}\left(p_{i} q_{i}\right)=\sqrt{\omega^{2}\left(p_{i}\right)+q_{i}^{2}}
$$

and

$$
\omega\left(p_{i}\right)=\sqrt{m_{j}^{2}+p_{i}^{2}}+\sqrt{m_{k}^{2}+p_{i}^{2}} \equiv \omega_{j}\left(p_{i}\right)+\omega_{k}\left(p_{i}\right) .
$$

The invariant volume element for three particles satisfying Eq. (2) can be written in terms of the corresponding volume element for the relative momenta as 


$$
\frac{d \vec{k}_{1}}{2 \omega_{1}\left(k_{1}\right)} \frac{d \vec{k}_{2}}{2 \omega_{2}\left(k_{2}\right)} \frac{d \vec{k}_{3}}{2 \omega_{3}\left(k_{3}\right)} \delta\left(\vec{k}_{1}+\vec{k}_{2}+\vec{k}_{3}\right)=\frac{\omega\left(p_{i}\right)}{8 W_{i}\left(p_{i} q_{i}\right) \omega_{i}\left(q_{i}\right) \omega_{j}\left(p_{i}\right) \omega_{k}\left(p_{i}\right)} d \vec{p}_{i} d \vec{q}_{i} .
$$

Therefore, if the single-particle states are normalized invariantly on the mass shell, i.e.,

$$
<\vec{k}_{i} \mid \vec{k}_{i}^{\prime}>=2 \omega_{i}\left(k_{i}\right) \delta\left(\vec{k}_{i}-\vec{k}_{i}^{\prime}\right),
$$

then the basis states $\mid \vec{p}_{i} \vec{q}_{i}>$ are normalized as

$$
<\vec{p}_{i} \vec{q}_{i} \mid \vec{p}_{i}^{\prime} \vec{q}_{i}^{\prime}>=\frac{8 W_{i}\left(p_{i} q_{i}\right) \omega_{i}\left(q_{i}\right) \omega_{j}\left(p_{i}\right) \omega_{k}\left(p_{i}\right)}{\omega\left(p_{i}\right)} \delta\left(\vec{p}_{i}-\vec{p}_{i}^{\prime}\right) \delta\left(\vec{q}_{i}-\vec{q}_{i}^{\prime}\right),
$$

and satisfy the completeness relation

$$
1=\int \frac{\omega\left(p_{i}\right)}{8 W_{i}\left(p_{i} q_{i}\right) \omega_{i}\left(q_{i}\right) \omega_{j}\left(p_{i}\right) \omega_{k}\left(p_{i}\right)} d \vec{p}_{i} d \vec{q}_{i}\left|\vec{p}_{i} \vec{q}_{i}><\vec{p}_{i} \vec{q}_{i}\right| .
$$

Introducing complete sets of basis states into the operator equations (6) and (7) one obtains the integral equations

$$
\begin{aligned}
<\vec{p}_{i} \vec{q}_{i} \mid \phi_{i}>= & G_{0}\left(W_{0} ; p_{i} q_{i}\right) \sum_{j \neq i} \int \frac{\omega\left(p_{i}^{\prime}\right)}{8 W_{i}\left(p_{i}^{\prime} q_{i}^{\prime}\right) \omega_{i}\left(q_{i}^{\prime}\right) \omega_{j}\left(p_{i}^{\prime}\right) \omega_{k}\left(p_{i}^{\prime}\right)} d \vec{p}_{i}^{\prime} d \vec{q}_{i}^{\prime} \\
& \times \frac{\omega\left(p_{j}\right)}{8 W_{j}\left(p_{j} q_{j}\right) \omega_{j}\left(q_{j}\right) \omega_{k}\left(p_{j}\right) \omega_{i}\left(p_{j}\right)} d \vec{p}_{j} d \vec{q}_{j}<\vec{p}_{i} \vec{q}_{i}\left|t_{i}\left(W_{0}\right)\right| \vec{p}_{i}^{\prime} \vec{q}_{i}^{\prime}> \\
& \times<\vec{p}_{i}^{\prime} \vec{q}_{i}^{\prime}\left|\vec{p}_{j} \vec{q}_{j}><\vec{p}_{j} \vec{q}_{j}\right| \phi_{j}>,
\end{aligned}
$$

and

$$
\begin{aligned}
<\vec{p}_{i} \vec{q}_{i}\left|t_{i}\left(W_{0}\right)\right| \vec{p}_{i}^{\prime} \vec{q}_{i}^{\prime}>= & <\vec{p}_{i} \vec{q}_{i}\left|V_{i}\right| \vec{p}_{i}^{\prime} \vec{q}_{i}^{\prime}>+\int \frac{\omega\left(p_{i}^{\prime \prime}\right)}{8 W_{i}\left(p_{i}^{\prime \prime} q_{i}^{\prime \prime}\right) \omega_{i}\left(q_{i}^{\prime \prime}\right) \omega_{j}\left(p_{i}^{\prime \prime}\right) \omega_{k}\left(p_{i}^{\prime \prime}\right)} d \vec{p}_{i}^{\prime \prime} d \vec{q}_{i}^{\prime \prime} \\
& \times<\vec{p}_{i} \vec{q}_{i}\left|V_{i}\right| \vec{p}_{i}^{\prime \prime} \vec{q}_{i}^{\prime \prime}>G_{0}\left(W_{0} ; p_{i}^{\prime \prime} q_{i}^{\prime \prime}\right)<\vec{p}_{i}^{\prime \prime} \vec{q}_{i}^{\prime \prime}\left|t_{i}\left(W_{0}\right)\right| \vec{p}_{i}^{\prime} \vec{q}_{i}^{\prime}>,
\end{aligned}
$$

where the matrix elements of the potential are given by [1]

$$
\begin{aligned}
<\vec{p}_{i} \vec{q}_{i}\left|V_{i}\right| \vec{p}_{i}^{\prime} \vec{q}_{i}^{\prime}>= & 8 \omega_{i}\left(q_{i}\right)\left[\frac{W_{i}\left(p_{i} q_{i}\right) \omega_{j}\left(p_{i}\right) \omega_{k}\left(p_{i}\right) W_{i}\left(p_{i}^{\prime} q_{i}\right) \omega_{j}\left(p_{i}^{\prime}\right) \omega_{k}\left(p_{i}^{\prime}\right)}{\omega\left(p_{i}\right) \omega\left(p_{i}^{\prime}\right)}\right]^{1 / 2} \\
& \times \delta\left(\vec{q}_{i}-\vec{q}_{i}^{\prime}\right) V_{i}\left(\vec{p}_{i}, \vec{p}_{i}^{\prime}\right),
\end{aligned}
$$

with $V_{i}\left(\vec{p}_{i}, \vec{p}_{i}^{\prime}\right)$ the usual Fourier transform of the potential

$$
V_{i}\left(\vec{p}_{i}, \vec{p}_{i}^{\prime}\right)=\frac{1}{(2 \pi)^{3}} \int d \vec{r} e^{i \vec{p}_{i} \cdot \vec{r}} V(r) e^{-i \vec{p}_{i}^{\prime} \cdot \vec{r}} .
$$

As shown in Ref. [1] these equations can be partial-wave projected into the basis $\mid p_{i} q_{i} ; \ell_{i} \lambda_{i}>_{L}$ where $\ell_{i}$ and $\lambda_{i}$ are the orbital angular momentum quantum numbers corresponding to the momenta $\vec{p}_{i}$ and $\vec{q}_{i}$, respectively, and $L$ is the total orbital angular momentum. Since the two-body interactions that we consider do not have tensor nor spin-orbit terms the spin is 
treated nonrelativistically by means of Racah coefficients in the same way as the isospin [1]. We solved the integral equations including all the configurations with $\ell_{i}$ and $\lambda_{i}$ up to 5 .

As has been already mentioned we will use a quark-quark potential of the type

$$
V_{q q}\left(\vec{r}_{i j}\right)=V_{C O N}\left(\vec{r}_{i j}\right)+V_{O G E}\left(\vec{r}_{i j}\right)+V_{O P E}\left(\vec{r}_{i j}\right)+V_{O S E}\left(\vec{r}_{i j}\right),
$$

where $\vec{r}_{i j}$ is the interquark distance $\left(\vec{r}_{i j}=\vec{r}_{i}-\vec{r}_{j}\right) . V_{C O N}$ is the confining potential taken to be linear,

$$
V_{C O N}\left(\vec{r}_{i j}\right)=-a_{c} \vec{\lambda}_{i} \cdot \vec{\lambda}_{j} r_{i j}
$$

$V_{O G E}$ is the one-gluon-exchange potential

$$
V_{O G E}\left(\vec{r}_{i j}\right)=\frac{1}{4} \alpha_{s} \vec{\lambda}_{i} \cdot \vec{\lambda}_{j}\left\{\frac{1}{r_{i j}}-\frac{1}{4 m_{q}^{2}}\left[1+\frac{2}{3} \vec{\sigma}_{i} \cdot \vec{\sigma}_{j}\right] \frac{e^{-r_{i j} / r_{0}}}{r_{0}^{2} r_{i j}}\right\},
$$

$\alpha_{s}$ is the effective quark-quark-gluon coupling constant, the $\lambda^{\prime} s$ are the $S U(3)$ color matrices, and the $\sigma^{\prime} s$ stand for the spin Pauli matrices. $V_{O P E}$ and $V_{O S E}$ are the pseudoscalar and scalar Goldstone-boson exchange potentials, given respectively by:

$$
\begin{gathered}
V_{\mathrm{OPE}}\left(\vec{r}_{i j}\right)=\frac{1}{3} \alpha_{c h} \frac{\Lambda^{2}}{\Lambda^{2}-m_{\pi}^{2}} m_{\pi}\left[Y\left(m_{\pi} r_{i j}\right)-\frac{\Lambda^{3}}{m_{\pi}^{3}} Y\left(\Lambda r_{i j}\right)\right] \vec{\sigma}_{i} \cdot \vec{\sigma}_{j} \vec{\tau}_{i} \cdot \vec{\tau}_{j}, \\
V_{\mathrm{OSE}}\left(\vec{r}_{i j}\right)=-\alpha_{c h} \frac{4 m_{q}^{2}}{m_{\pi}^{2}} \frac{\Lambda^{2}}{\Lambda^{2}-m_{\sigma}^{2}} m_{\sigma}\left[Y\left(m_{\sigma} r_{i j}\right)-\frac{\Lambda}{m_{\sigma}} Y\left(\Lambda r_{i j}\right)\right],
\end{gathered}
$$

where $m_{\pi}\left(m_{\sigma}\right)$ is the pion (sigma) mass, $\alpha_{c h}$ is the chiral coupling constant related to the $\pi N N$ coupling constant through $\alpha_{c h}=\left(\frac{3}{5}\right)^{2} \frac{g_{\pi N N}^{2}}{4 \pi} \frac{m_{\pi}^{2}}{4 m_{N}^{2}}, \Lambda$ is a cutoff parameter, and $Y(x)$ is the standard Yukawa function $Y(x)=e^{-x} / x$

The one-gluon-exchange potential shown in Eq. (21) contains a smeared-out delta function with smearing parameter $r_{0}$. As it has been shown in Refs. [1,7] this smeared delta function has a very important effect on the baryon spectrum. For example, in the case of the nonrelativistic theory [7], if $r_{0} \leq 0.1 \mathrm{fm}$ the mass of the nucleon collapses to very large negative values. On the other hand, in the case of the relativistic theory the collapsing of the nucleon mass occurs for $r_{0} \leq 0.45 \mathrm{fm}$ due to the fact that in this case the three-body propagator falls down more slowly in momentum space [1].

The behavior of the solution of the three-body equations in the presence of a delta function will have also important consequences when one includes the one-pion-exchange interaction. As can be seen in Eq. (22) the one-pion-exchange potential consists of a Yukawa interaction with a range equal to the inverse of the pion mass plus a smearedout delta function with smearing parameter $1 / \Lambda$. Therefore, also in this case the mass of the nucleon will collapse if $1 / \Lambda \leq 0.1 \mathrm{fm}$ in the case of the nonrelativistic theory and if $1 / \Lambda \leq 0.45 \mathrm{fm}$ in the case of the relativistic one. Thus, the cutoff parameter $\Lambda$ must be smaller than $\sim 10 \mathrm{fm}^{-1}$ in the case of the nonrelativistic theory and $\sim 2.2 \mathrm{fm}^{-1}$ in the case of the relativistic one. This is due to the fact that the nonrelativistic propagator falls 
down as $1 / k^{2}$ while the relativistic one does it as $1 / k$. This is, for example, the reason why the semirelativistic solutions of Ref. [11] collapse when a scalar and an one-gluonexchange potential are added to the pseudoscalar interaction. They include a strong onegluon exchange attraction, $1 / \Lambda_{g} \sim 0.20 \mathrm{fm}$, over the strong attractive interaction already provided by the one-pion exchange, $1 / \Lambda_{\pi} \sim 0.29 \mathrm{fm}$. This makes evident the risk of replacing the nonrelativistic kinetic energy by the relativistic one when the details of the short-range part of the potential are not well known.

Let us first try to illustrate how the combined effect of relativistic kinematics and the onepion-exchange interaction gives rise to the inversion between the first positive- and negativeparity nucleon excited states. For this purpose we consider the quark-quark interaction given in Eq. (19) with $V_{O S E}=0$ and the set of parameters of Table I. The obtained spectrum is shown in Fig. 1. As can be seen the positive parity $N^{*}(1440)$, Roper resonance, lies below the negative parity $N^{*}(1535)$ state, while the remaining states of the spectrum are correctly reproduced. The same effect has been observed in a nonrelativistic study (see Fig. 4 of Ref. [7]) at the price of loosing the description of the remaining states of the spectrum. It is only when relativistic kinematics is introduced, that the inversion between positive- and negative-parity excitations of the nucleon is achieved with an overall good description of the rest of the spectrum. The relativistic kinematics alone decreases a lot the excitation energy of the Roper resonance with respect to the first excited state of negative parity as compared to a nonrelativistic description [3]. The Roper resonance is particularly sensitive to the form of the kinetic energy [3]. Once this energy difference has been decreased, the one-pion exchange is able to produce the desired inversion without destroying the description of the rest of the spectrum.

Chiral symmetry requires also the presence of an scalar Goldstone boson exchange. Although in a perturbative description its effect would be minor, we have calculated the spectrum obtained when one takes also into account the scalar interaction given by Eq. (23) with the same set of parameters shown in Table I. The results are presented in Fig. 2. As one can see, the agreement with the experimental data is much better than in the previous case. It reduces the splitting between the $J^{P}=1 / 2^{+}$states $N^{*}(1440)$ and $N^{*}(1710)$, and increases the distance between the ground and first excited states of negative parity. On the other hand, the $\Delta(1232)$ comes out somewhat higher although we have not made any effort to fine tune the parameters of the model, our main objective being the effect on the relative position of the positive and negative parity nucleon states.

The reverse of the ordering achieved by means of the pion exchange is due to a similar mechanism than the one proposed in Ref. [6] by means of a scalar three-body force. Such a force is very short-ranged and in the limit of zero range it acts only for states whose wave functions do not cancel at the origin. It therefore affects the nucleon and its radial excitations, while producing essentially no effect for states with mixed symmetry (negative parity nucleon excited states). Thus, this force, if it is chosen attractive, explains why the Roper resonance is lower than the negative parity excited states in the nucleon sector.

In the case of the chiral pseudoscalar interaction, its $(\vec{\sigma} \cdot \vec{\sigma})(\vec{\tau} \cdot \vec{\tau})$ structure gives attraction for symmetric spin-isospin pairs and repulsion for antisymmetric ones (a quite distinctive feature since the color-magnetic part of the OGE gives similar contributions in both cases). This lowers the position of the first nucleon radial excitation $\left[N^{*}(1440)\right]$ with a completely symmetric spin-isospin structure with regard to the first negative parity state $\left[N^{*}(1535)\right]$, 
with a mixed symmetry wave function in spin-isospin space. Due to the decreasing of the excitation energy of the Roper resonance induced by the relativistic kinematics, the pseudoscalar force is able to solve the discrepancy between usual two-body potential models (the predicted relative energy positions of the Roper and the first negative parity appear inverted) and experiment.

A final comment regarding the one-gluon-exchange interaction is in order. As has been explained this potential alone is not able to reverse the order of the Roper resonance and the first $N\left(1 / 2^{-}\right)$state [1]. However, we did not find any limit to the strength of this potential for a correct description of the spectrum once relativistic kinematics and the one-pion exchange are introduced. The parameters we have used for the one-gluon exchange are the standard values used to study the $N N$ interaction $\alpha_{s} \sim 0.4-0.5$. One could imagine that a better fit to the baryon spectrum could be obtained by decreasing the strength of the one-gluon exchange, what could provide with a bigger inversion as explained in the previous paragraph. However, the effect obtained is exactly the opposite one. In Fig. 2 the mass difference between the Roper resonance and the first $N\left(1 / 2^{-}\right)$state is $-70 \mathrm{MeV}$. If we recalculate the same spectrum using $\alpha_{s}=0.4$ instead of $\alpha_{s}=0.5$, there is a general decreasing of the mass of all the states, the $\Delta\left(3 / 2^{+}\right)$appears at $270 \mathrm{MeV}$ and the Roper resonance appears at $465 \mathrm{MeV}$, that could be cured increasing the slope of the confining potential. However, the mass difference between the Roper resonance and the first $N\left(1 / 2^{-}\right)$state is reduced to $-31 \mathrm{MeV}$. Therefore, once the relativistic kinematics and the one-pion exchange are considered the effect of the one-gluon exchange cannot be estimated qualitatively, because it generates repulsion on both states but the strength depends on the wave function, and therefore on the other interactions considered. This observation reinforces the notion that the one-gluon-exchange force is necessary for the description of the $N N$ interaction [12].

As a summary, we have shown that the relative position of the positive and negative parity low-energy states is determined basically by the relativistic kinematics together with the one-pion-exchange interaction. A great improvement is obtained by adding the chiral partner of the pion, the sigma meson. We did not find any constrain to the strength of the one-gluon exchange potential. Finally, let us stress that the replacement of the nonrelativistic by the relativistic kinematics for short-range attractive interactions has to be done with a careful study of the short-range part of the potential in the relativistic approach.

\section{ACKNOWLEDGMENTS}

We are specially grateful to Dr. B. Desplanques for calling our attention on the problem raised in this work and giving us a lot of technical and practical information. We also thank to Dr. P. González for enlightening discussion. This work has been partially funded by COFAA-IPN (México), by Ministerio de Ciencia y Tecnología under Contract No. BFM2001-3563 and by Junta de Castilla y León under Contract No. SA-109/01. 


\section{REFERENCES}

[1] H. Garcilazo, Phys. Rev. C 67, 055203 (2003).

[2] J.L. Basdevant and S. Boukraa, Z. Phys. C 30, 103 (1986).

[3] J. Carlson, J. Kogut, and V.R. Pandharipande, Phys. Rev. D 27, 233 (1983).

[4] B. Silvestre-Brac and C. Gignoux, Phys. Rev. D 32, 743 (1985).

[5] N. Isgur, Phys. Rev. D 62, 054026 (2000).

[6] B. Desplanques, C. Gignoux, B. Silvestre-Brac, P. González, J. Navarro, and S. Noguera, Z. Phys. A 343, 331 (1992).

[7] H. Garcilazo, A. Valcarce, and F. Fernández, Phys. Rev. C 63, 035207 (2001); ibid 64, 058201 (2001).

[8] L.Ya. Glozman and D.O. Riska, Phys. Rep. 268, 263 (1996).

[9] A. Valcarce, A. Faessler, and F. Fernández, Phys. Lett. B 345, 367 (1995).

[10] B. Juliá-Díaz, J. Haidenbauer, A. Valcarce, and F. Fernández, Phys. Rev. C 65, 034001 (2002).

[11] M. Furuichi and K. Shimizu, Phys. Rev. C 65, 025201 (2002).

[12] C. Nakamoto and H. Toki, Prog. Theor. Phys. 99, 1001 (1998). 


\section{FIGURES}

FIG. 1. Nonstrange baryon spectrum for the interaction given in Eq. (19) with $V_{O S E}=0$ and the set of parameters of Table I. Masses are given relative to the nucleon mass.

FIG. 2. Nonstrange baryon spectrum for the interaction given in Eq. (19) and the set of parameters of Table I. Masses are given relative to the nucleon mass. 


\section{TABLES}

TABLE I. Quark model parameters for the calculation of Figs. 1 and 2.

\begin{tabular}{cc}
\hline \hline$m_{q}(\mathrm{MeV})$ & 313 \\
$\alpha_{s}$ & 0.50 \\
$a_{c}\left(\mathrm{MeV} \cdot \mathrm{fm}^{-1}\right)$ & 110.0 \\
$\alpha_{c h}$ & 0.0269 \\
$r_{0}(\mathrm{fm})$ & 0.74 \\
$m_{\sigma}\left(\mathrm{fm}^{-1}\right)$ & 3.42 \\
$m_{\pi}\left(\mathrm{fm}^{-1}\right)$ & 0.7 \\
$\Lambda\left(\mathrm{fm}^{-1}\right)$ & 2.0 \\
\hline \hline
\end{tabular}




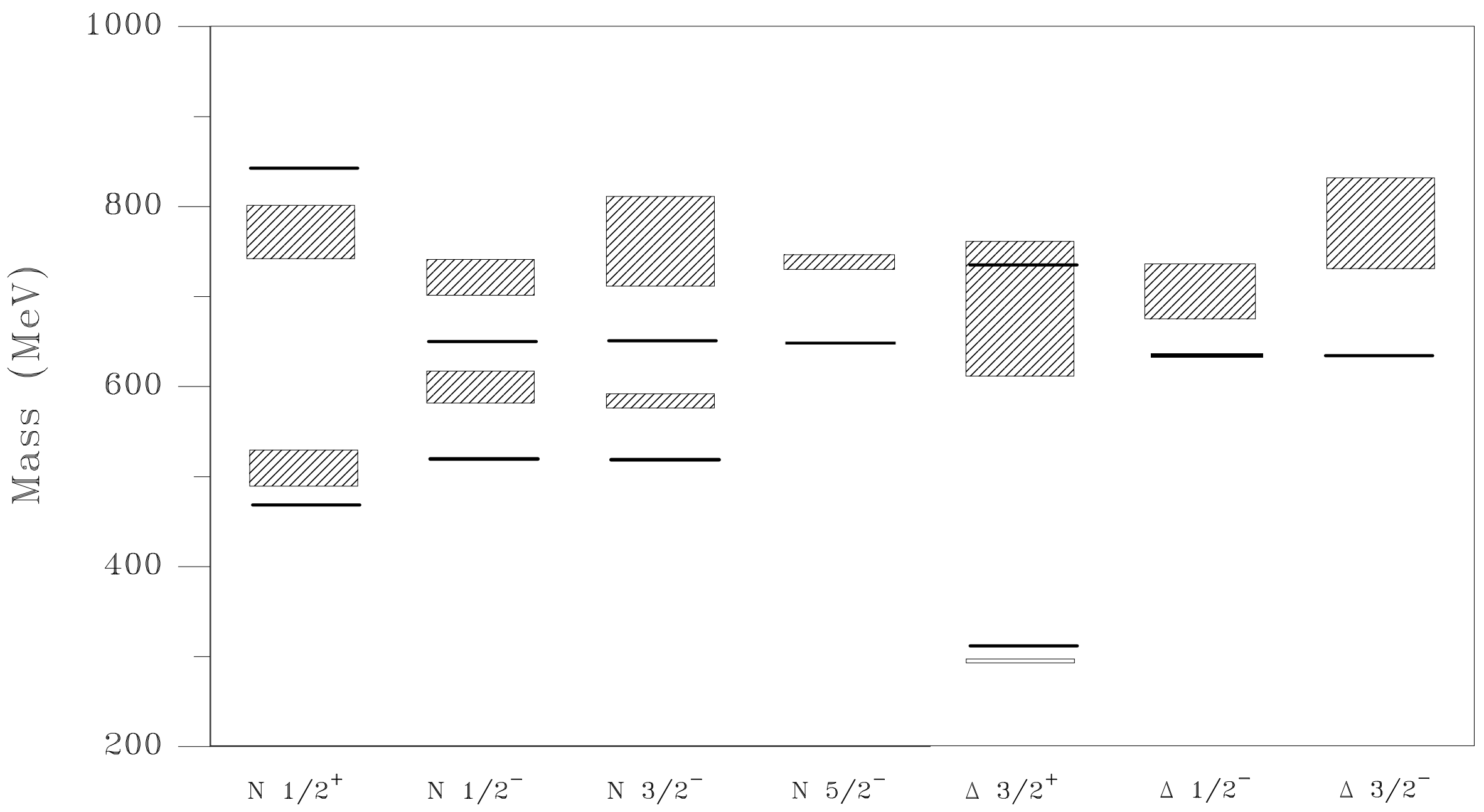

Figure 1 


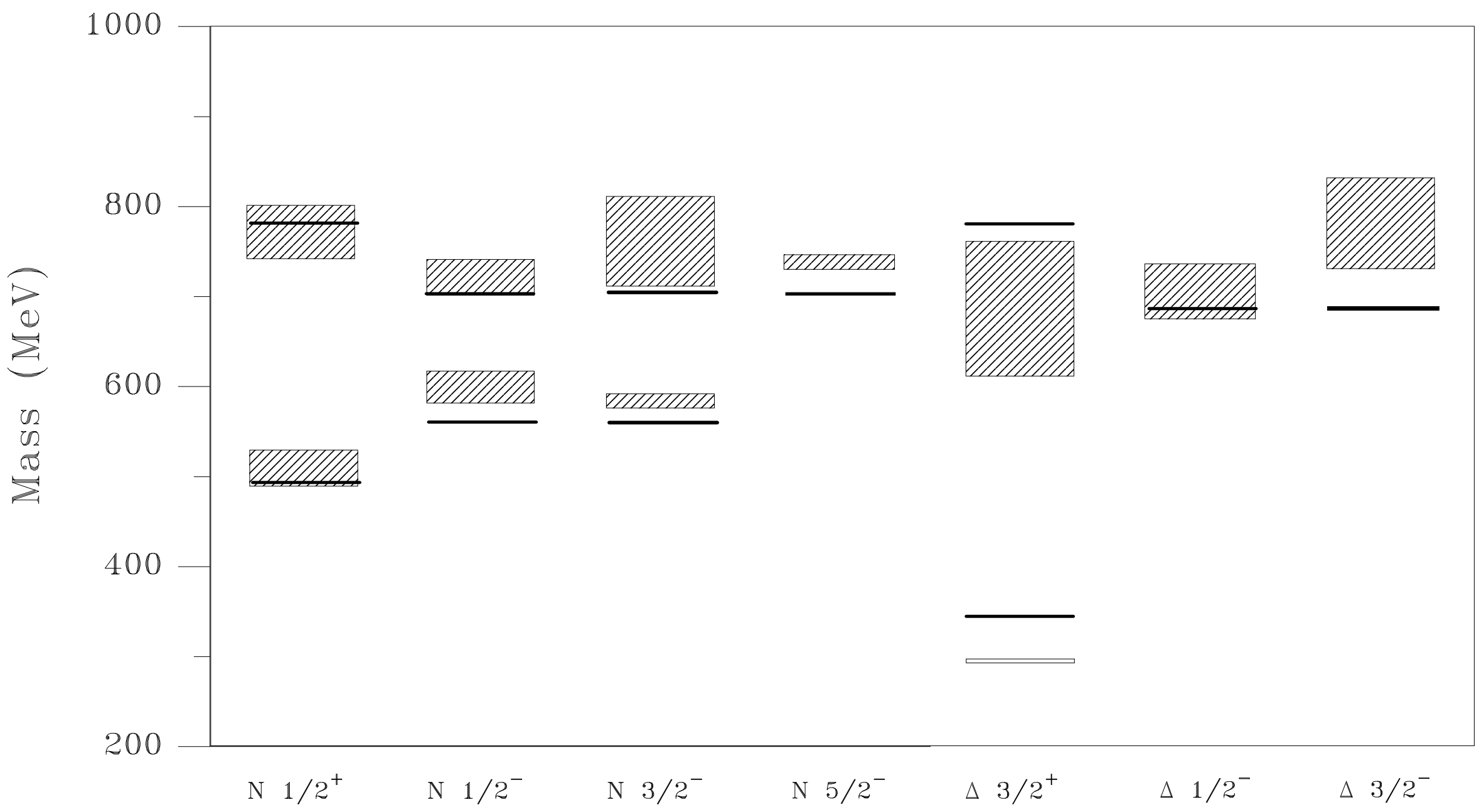

Figure 2 